\title{
A new cam-geared mechanism for exact path generation
}

\author{
Ren-Chung SOONG* \\ * Department of Mechanical and Automation Engineering, Kao Yuan University \\ 1821, Jhongshan Rd., Lujhu Dist, Kaohsiung City, 82151, Taiwan, R.O.C. \\ E-mail: t30004@jcc.kyu.edu.tw
}

Received 2 March 2015

\begin{abstract}
A new cam-geared mechanism, consisting of a cam-follower and elementary planetary gear train is proposed for exact path generation. The formation and mobility of the proposed mechanism are illustrated in detail, in addition to inverse kinematic analysis of the design and the design procedure. The advantages of the new design include its simple, compact structure, simple design procedure and no numbers limitation of precision point. This new mechanism is suitable for generating diverse continuous curve paths and symmetric curve paths with an intersection. Examples are provided to show the feasibility and effectiveness of this proposed method.
\end{abstract}

Key words : Cam-geared mechanism, Cam-follower mechanism, Elementary planetary gear train, Exact path generation

\section{Introduction}

Path generation is defined as the creation of a trajectory of a specific point in a plane such that it moves along a prescribed path. Path generation can typically be classified into two types: point-to-point path generation and exact path generation. In point-to-point generation, the coupler point specifies several points on the prescribed path (i.e., the path between any two precision points is not prescribed). Over the past several decades, there have been numerous studies on point-to-point path generation and optimal synthesis methods, including the techniques of unconstrained nonlinear least-squares (Angeles, et al., 1988), Fourier descriptors (Chuenchom and Kota, 1997), adjustable dyads (Ullah and Kota, 1997), orientation structural error (Zhou and Cheung, 2001), neural networks (Vasiliu and Yannou, 2001), exact gradient determination (Sancibrian, et al., 2006), shape invariants (Buskiewicz, 2010), and genetic or differential evolution algorithms (Cabrera, et al., 2002, Laribi, et al., 2004, Lin, 2010, Matekar and Gogate, 2012). Several methods based on the point-to-point technique have been applied to multi-path generation (Zhou and Ting, 2002, Russell and Sodhi, 2005, Peng and Sodli, 2015, Chanekar and Ghosal, 2013).

Exact path generation uses a coupler point to specify the entire prescribed path or many points along the prescribed path. Compared with point-to-point path generation, fewer studies have used this approach in path generation design. However, linkage and cam-linkage mechanisms, especially, as well as geared linkage mechanisms, are commonly described using exact plane path generation techniques. Key and Haws (1975) proposed a cam-link mechanism to generate precisely the desired path using a fixed cam to adjust the length of the side link in a four-bar linkage configuration. Singh and Kohli (1981) combined the complex loop closure method and envelope theory to attain the exact path or motion generation for the synthesis of combined cam-linkage systems. Shimojima et al. (1983) developed a synthesis method for straight line and L-shaped path generation, using fixed pivot positions as adjustable parameters. Ye and Smith (2005) presented an analytical method for a combined cam-linkage mechanism with an oscillating roller follower for tracing a prescribed path. Mundo et al. (2006) presented an optimal synthesis method for cam-linkage mechanisms with one or more disk cams for exact path generation. Gatti and Mundo (2007) proposed a technique for optimal synthesis of cam-linkage mechanisms with two disk cams for exact rigid-body guidance. Soong and Wu (2009) demonstrated a variable coupler curve-generating mechanism by controlling the angular displacement of the driving link and adjusting the link length of the fixed link. Mundo et al. (2009) proposed a synthesis method for five-bar linkage mechanisms with a pair of non-circular gears to generate exact prescribed paths using a genetic algorithm. Zhou (2009) used adjustable four-bar linkages for the precise generation of continuous paths by adjusting the pivot 
location of the driven side link. Soong and Chang (2011) applied the inverse cam design to obtain the desired output motion or exact function generation by varying the link length of the driving link for single degree-of-freedom (DOF) linkage mechanisms. Soong (2013) proposed a mechanism with a linear input link and a rotational input link for exact path generation by controlling the instantaneous position of two input links.

In this paper, we proposed a new design method for cam-geared mechanisms to generate the prescribed exact paths. The formation of the proposed mechanism is presented, in addition to inverse kinematic analysis of the design and the design procedure. Examples are provided to demonstrate the feasibility and effectiveness of this design method.

\section{New exact path generation mechanism}

The new one degree of freedom (1-DOF) cam-geared mechanism, driven by a rotational input link, is shown in Fig. 1. The different links are distinguished by colors and numbers in Fig 1.

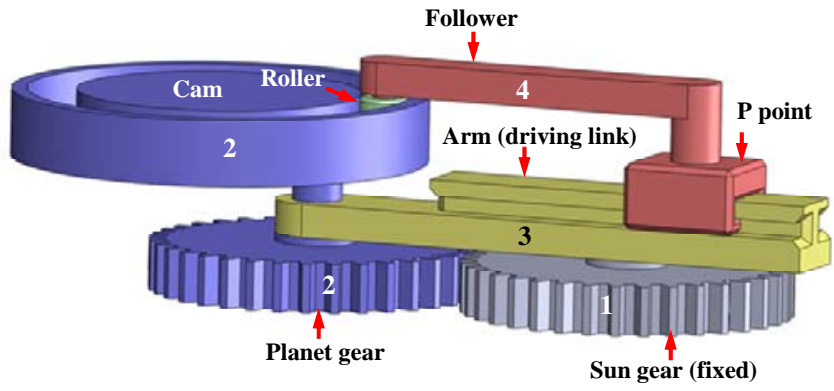

(a)

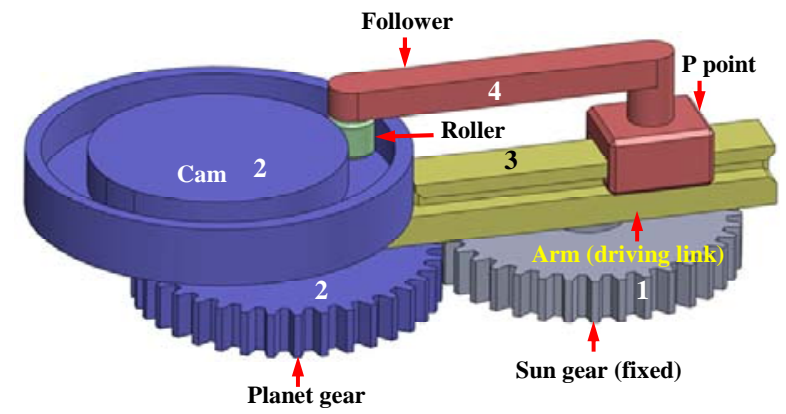

(b)

Fig. 1 Proposed exact path generation mechanism

This proposed mechanism is a four-bar cam-geared mechanism with two revolute joints, a sliding joint, a cam joint, and a gear joint. In this mechanism, the elementary planetary gear train consists of links 1 through 3 . Link 1 is a sun gear and is fixed, while link 3 is an arm and serves as the input link. Link 2 is a planet gear. The cam is attached to link 2, roller, and link 4 (which moves along a linear guide on link 3), forming a cam-follower mechanism. If the gear ratio between the sun gear and the planet gear is equal to 1, the point p on the output link (link 4), as shown in Fig. 1, can generate a prescribed path while the input link completes a single motion cycle. According to Grubler-Kutzbach criteria for planar mechanisms, $\mathrm{DOF}=3(\mathrm{~N}-1)-\sum \mathrm{J}_{\mathrm{i}} \mathrm{C}_{\mathrm{pi}}, \mathrm{N}$ denotes the numbers of link, $\mathrm{J}_{\mathrm{i}}$ denotes the numbers of $\mathrm{i}$ type joint and $\mathrm{C}_{\mathrm{pi}}$ denotes the degrees of constraint of i type joint. Since the proposed mechanism has four links, two revolute joints, a sliding joint, a cam joint, and a gear joint, the number of DOF of the proposed mechanism can be computed as follow:

$\mathrm{DOF}=3 *(4-1)-(2+1) * 2-(1+1) * 1=1$.

\section{Kinematic analysis}

Figure 2 shows the coordinate systems of the proposed 1-DOF five-bar cam-geared mechanism. The point $\mathrm{O}$ and point $\mathrm{D}$ are the origins of fixed coordinates and moving coordinates, respectively. We assume that the gear ratio between the sun gear and planet gear is equal to 1 . In order to minimize the largest translational displacement of the link 4 (follower) while generating prescribed paths, the coordinate values of point A (center of the sun gear), $\left(x_{A}, y_{A}\right)$, which has the average shortest distance to each point on the prescribed path, as shown in Figure 2, can be obtained by a process of optimization as follow:

$$
\text { Minimize } \operatorname{dis}=\sum_{i=1}^{n} \sqrt{\left(x_{p i}-x_{A}\right)^{2}+\left(y_{p i}-y_{A}\right)^{2}}
$$

Where $x_{p i}$ and $y_{p i}$ denote the coordinate values of the ith point on prescribed path in $\mathrm{X}$ and $\mathrm{Y}$ directions, respectively. The $\mathrm{n}$ presents the number of precision points on prescribed path.

Once $\left(x_{A}, y_{A}\right)$ are determined, the distance between center of the sun gear and the ith point on prescribed path, $r_{p i}$, can be expressed as follow: 


$$
r_{p i}=\sqrt{\left(x_{p i}-x_{A}\right)^{2}+\left(y_{p i}-y_{A}\right)^{2}}
$$
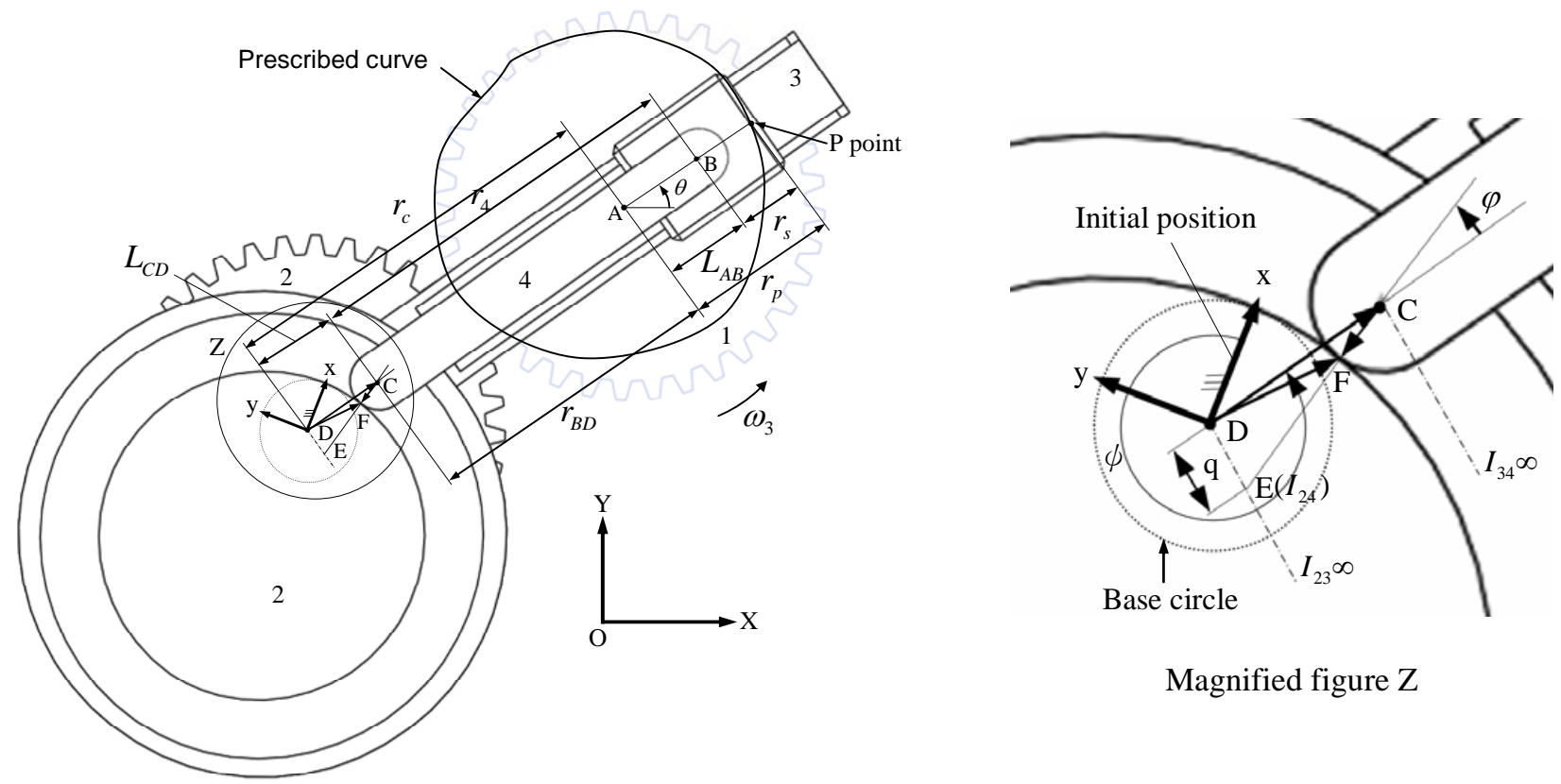

Magnified figure Z

Fig. 2 Coordinate system of the exact path generation mechanism

In order to minimize the maximum displacement of the follower (link 4) shown in Fig. 2 in a motion cycle, we let $r_{s}=r_{p \min }$.

Then, the maximum displacement of link 4 (follower) in a motion cycle, $r_{A \max }$, can be computed as follow:

$$
r_{A \max }=r_{p \max }-r_{p \min }
$$

Where $r_{s}, r_{p \max }$ and $r_{p \text { min }}$ represent the distance between point $\mathrm{B}$ and point $\mathrm{P}$ and the maximum and the minimum distance between point A and points on the prescribed path, respectively, as shown in Figure 2.

The instantaneous angular position of driving link corresponding to the ith point on the prescribed path, $\theta_{i}$, can be solved as follow:

$$
\theta_{i}=\tan ^{-1}\left(y_{p i} / x_{p i}\right)
$$

Since the cam 2 and link 4 revolute with link 3 (arm), the cam rotates around the center of planet gear and the link 4 translates along the center line of the link 3, simultaneously, in any instant, we can regard the combination of cam 2 , link4 (follower) and link3 as a cam with a translating roller follower mechanism.

A coordinate system $(x, y)$ is fixed on the cam 2 as shown in Figure 2. The instantaneous angular position of cam (link 2) relative to the coordinate system $(\mathrm{x}, \mathrm{y})$ corresponding to the ith point on the prescribed path, $\phi_{i}$, can be obtained as follow:

$$
\phi_{i}=360^{\circ}-\theta_{i}
$$

Then, the distance between point A and point B as shown in Figure 2, the instantaneous linear displacement of link 4 (follower) corresponding to the ith point on the prescribed path, $L_{A B i}$, can be obtained as follow:

$$
L_{A B i}=r_{p i}-r_{s}
$$

Once $L_{A B i}$ is determined, the distance between point $\mathrm{D}$ and point $\mathrm{C}$ as shown in Figure 2, $L_{C D i}$, can be expressed as follow:

$$
L_{C D i}=r_{c}-r_{4}+L_{A B i}
$$


Where $r_{c}$ denotes the distance between center of sun gear( point A) and center of planet gear ( point D) and $r_{4}$ denotes length of the link 4, as shown in Figure 2. Note that the value of $L_{C D i}$, namely, is summation of the radius of base circle of the cam and the radius of roller when the value of $L_{A B i}$ is equal to zero.

The three instant centers of link 2, link 3 and link 4, $I_{23}, I_{24}$ and $I_{34}$, are shown in Figure 2, respectively. The point $E$ presents instant center $I_{24}$ and let $\mathrm{DE}=\mathrm{q}$, then the speed of point $\mathrm{E}$ on link 2 (cam) relative to link $3, v_{E}$ can be presented as follow:

$v_{E}=q w_{2 / 3}=q w_{3}$

Where $w_{2 / 3}$ and $w_{3}$ denote the angular velocity of link 2 relative to link 3 and the angular velocity of link 3 (driving link), respectively.

The speed of point $\mathrm{E}$ on link 4 (follower) relative to link 3, $v_{E}$ can be expressed as follow:

$v_{E}=\frac{d L_{D C}(\phi)}{d t}=\frac{d L_{C D}(\phi)}{d \phi} \frac{d \phi}{d t}=\frac{d L_{C D}(\phi)}{d \phi} w_{2 / 3}=\frac{d L_{C D}(\phi)}{d \phi} w_{3}$

Comparing Equation (8) and Equation (9), we can obtain as

$q=\frac{d L_{C D}(\phi)}{d \phi}=v_{C D}(\phi)$

Where $v_{C D}(\phi)$ presents the speed of link 4 relative to link 3 .

Due to the instantaneous linear displacement of Link 4 (the follower) relative to link 3 , to compute the pressure angles of the cam, $L_{C D i}$ is expressed as a function of $\phi_{i}$ by a polynomial curve as follows:

$L_{C D i}\left(\phi_{i}\right)=c_{0}+c_{1} \phi_{i}+c_{2} \phi_{i}^{2}+c_{3} \phi_{i}^{3}+\cdots+c_{n-1} \phi_{i}^{n-1}$

where $C_{0}, C_{1}, \cdots, C_{n}$ are constants, and $n$ represents the number of precision points on the prescribed path.

Thus, the velocity of Link 4 (the follower), relative to the coordinate system $(x, y)$ corresponding to the $i^{\text {th }}$ point on the prescribed path, $v_{C D i}\left(\phi_{i}\right)$, is given by

$v_{C D i}\left(\phi_{i}\right)=c_{1}+2 c_{2} \phi_{i}+3 c_{3} \phi_{i}^{2}+\cdots+(n-1) c_{n-2} \phi_{i}^{n-2}$

The pressure angle of the cam $\varphi_{i}$ corresponding to the $i^{\text {th }}$ point on the prescribed path shown in Figure 2, is as follows:

$\varphi_{i}=\tan ^{-1}\left(\frac{v_{C D i}\left(\phi_{i}\right)}{L_{C D i}\left(\phi_{i}\right)}\right)$.

Once the pressure angles of the cam are determined, the vector equation of the cam profile relative to the moving coordinate system $(x, y)$ can be written as

$$
\overrightarrow{D F}=\overrightarrow{D C}+\overrightarrow{C F}
$$

Then, the coordinate values of the cam profile relative to the moving coordinate system $(x, y),\left(x_{c p i}, y_{c p i}\right)$, can be written as

$$
\begin{aligned}
& x_{c p i}=L_{C D i} \cos \left(\phi_{i}\right)-r_{f} \cos \left(\phi_{i}-\varphi_{i}\right) \\
& y_{c p i}=L_{C D i} \sin \left(\phi_{i}\right)-r_{f} \sin \left(\phi_{i}-\varphi_{i}\right)
\end{aligned}
$$

Where $r_{f}$ presents the radius of the roller.

\section{Design procedure}

To simplify the proposed method for easy application, a design procedure was proposed for exact path generation, as illustrated in Fig. 3. 


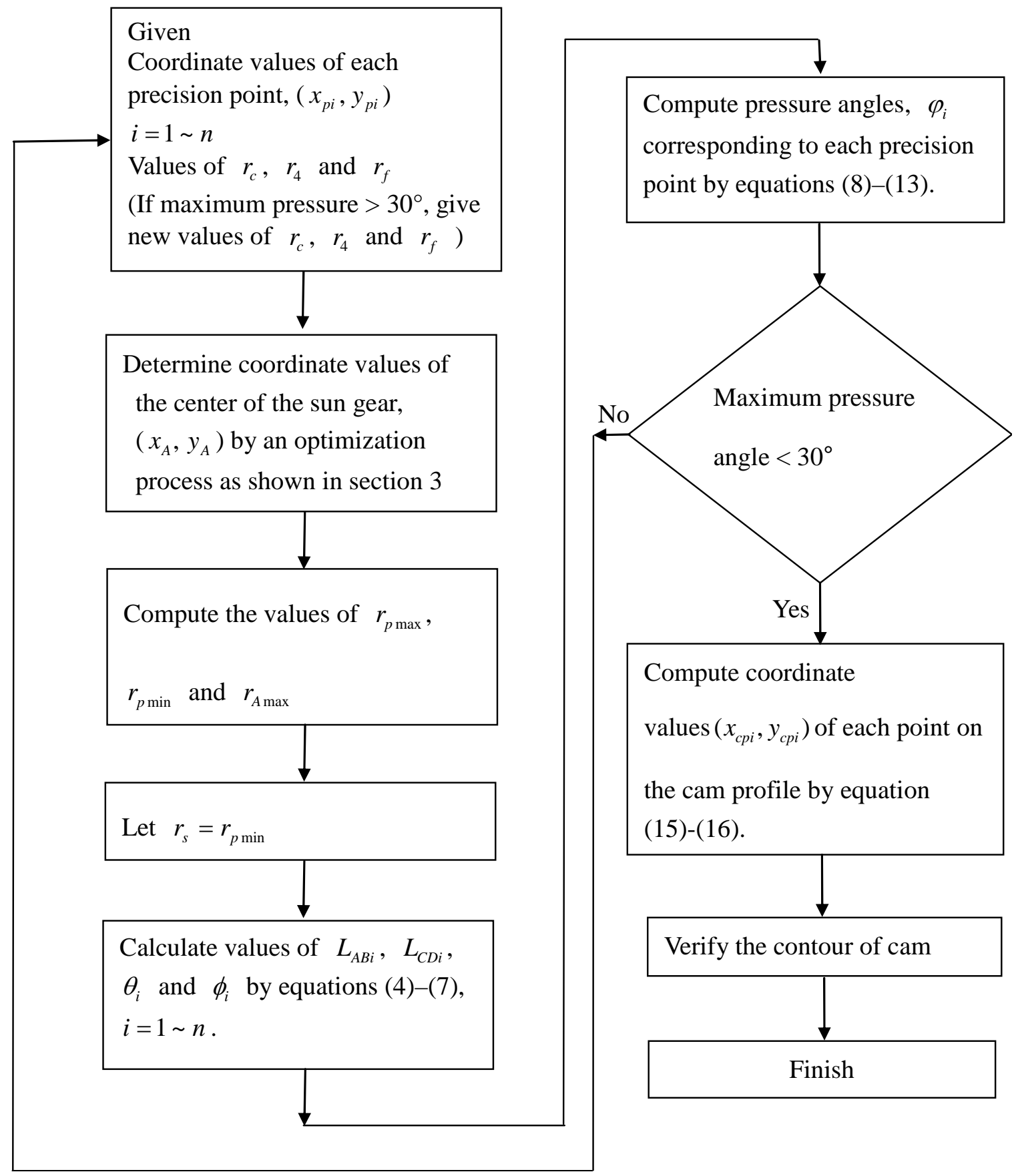

Fig. 3 Design procedure

\section{Design examples}

In the following examples, we demonstrate how the proposed mechanism shown in Fig. 1 generates different prescribed paths. In order to obtain the coefficients in Equation (11), we divide the prescribed exact path into several sections each section including 5 or 6 precision points.

\section{Example 1.}

In this example, we will generate a prescribed elliptically curved path, as shown in Fig. 4. The coordinate values of Point A, $\left(x_{A}, y_{A}\right)$, the maximum and minimum distances between Point A and points on the prescribed path, $\left(r_{p \text { max }}\right.$, $r_{p \text { min }}$ ), and the values of $r_{c}, r_{4}$, and $r_{f}$ are shown in Table 1. Figures 5-7 show the profile of the generated cam, the displacement of link4 (follower) and configurations of the proposed mechanism corresponding to the prescribed path, respectively. 


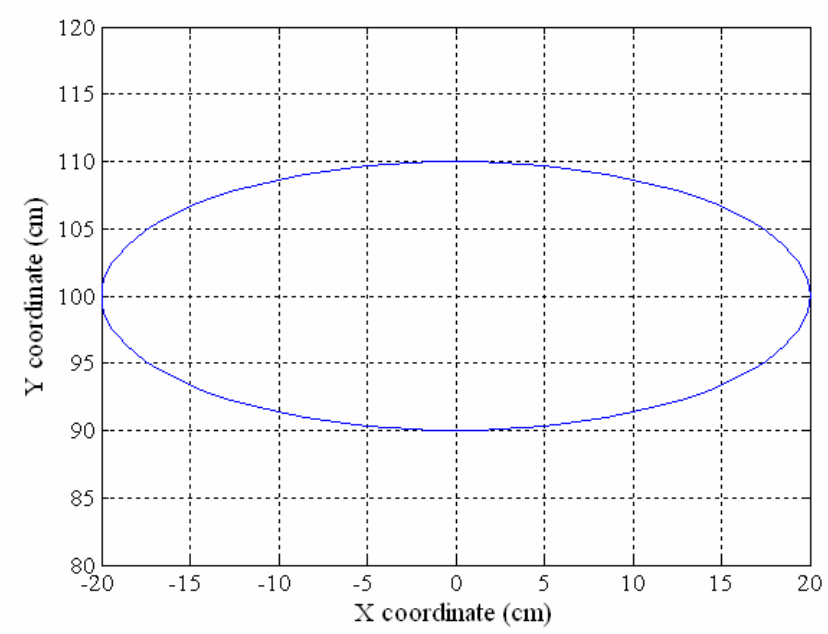

Fig. 4. Prescribed elliptically curved path for Example 1.

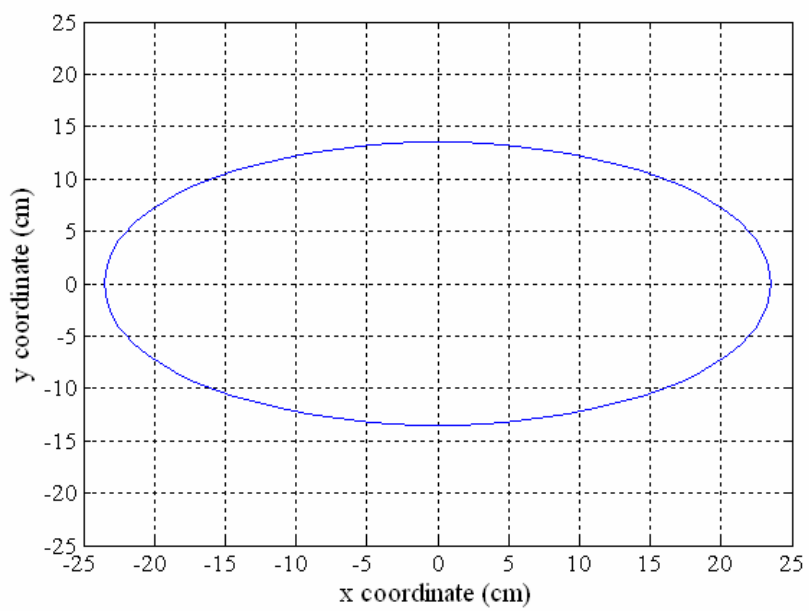

Fig. 5. Generated cam profile for Example 1.

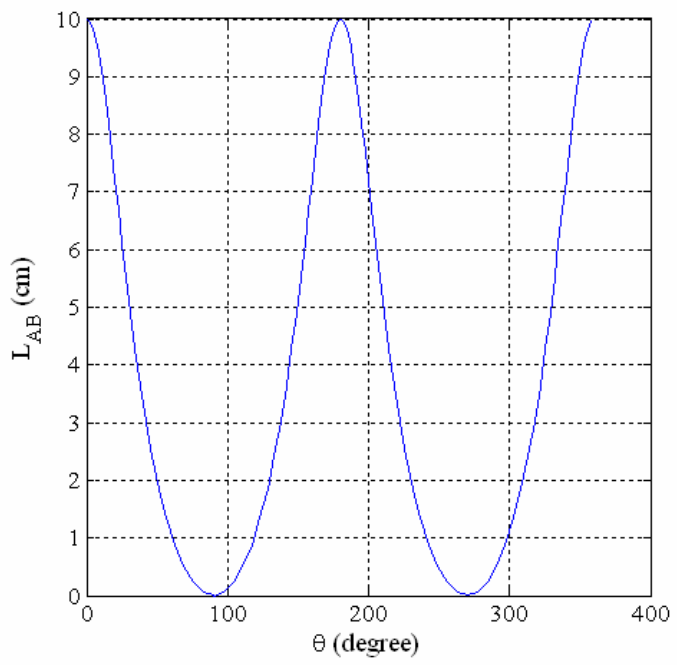

Fig. 6 The displacement of link 4 (follower) for example 1 


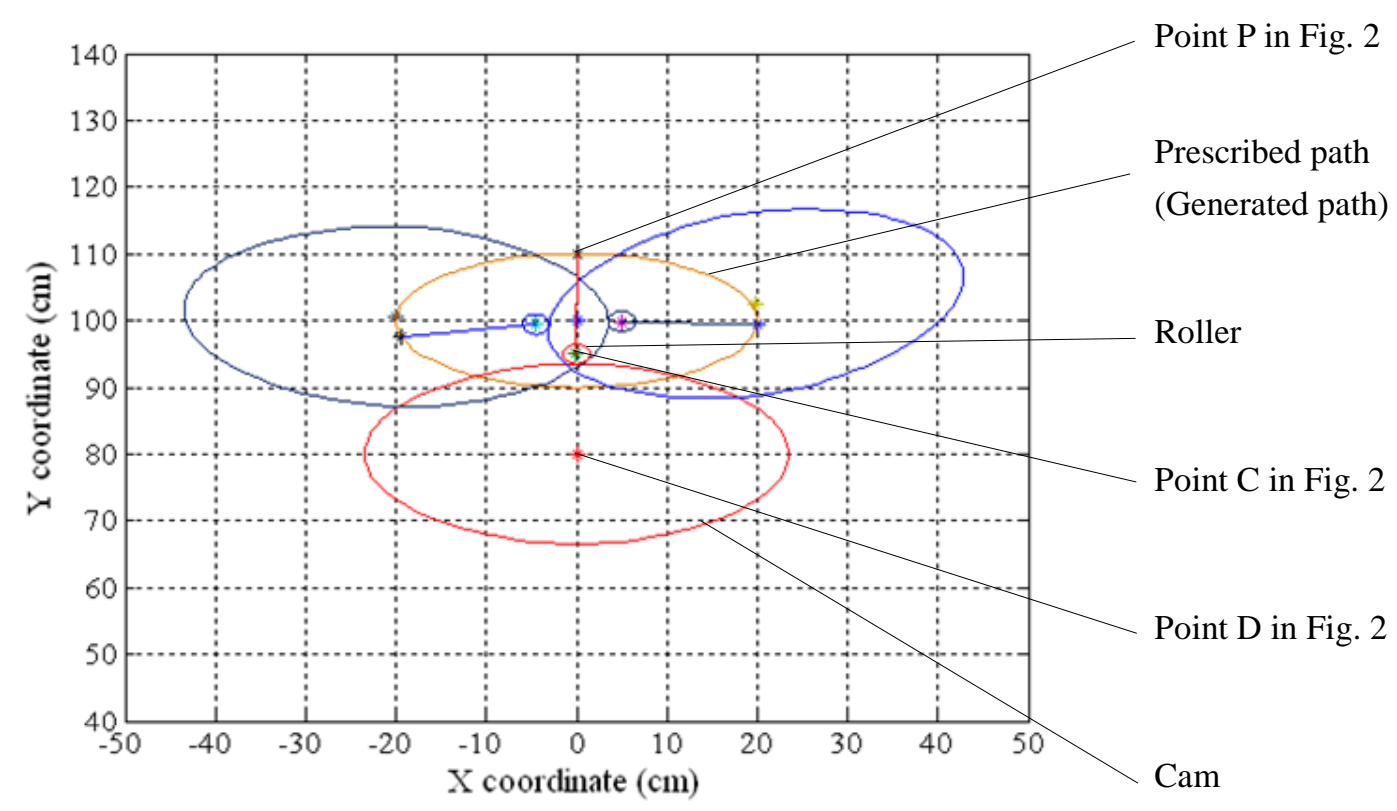

Fig. 7. Configurations corresponding to three precision points for Example 1.

Table 1. Design variables for Example 1 in centimeters $(\mathrm{cm})$.

\begin{tabular}{ccccccc}
\hline$r_{c}$ & $r_{4}$ & $r_{f}$ & $x_{A}$ & $y_{A}$ & $r_{p \max }$ & $r_{p \min }$ \\
\hline 20 & 5 & 1.5 & 0 & 100 & 20 & 10 \\
\hline
\end{tabular}

The results of this example show that the shape and size of the generated cam profile were very similar to the prescribed path and satisfied the condition of a maximum pressure angle less than $30^{\circ}$. The results of this example also suggest that the proposed mechanism is suitable for generating continuous curve paths.

\section{Example 2.}

In this example, we will generate a prescribed path as shown in Fig. 8, with the coordinates of each precision point listed in Table 2. The coordinate values of Point A, $\left(x_{A}, y_{A}\right)$, the maximum and the minimum distances between Point A and points on the prescribed path, $\left(r_{p \max }, r_{p \min }\right)$, and the values of $r_{c}, r_{4}$, and $r_{f}$ are given in Table 3. Figures 9-11 show the profile of the generated cam, the displacement of link4 (follower) and configurations of the proposed mechanism corresponding to the prescribed path, respectively.

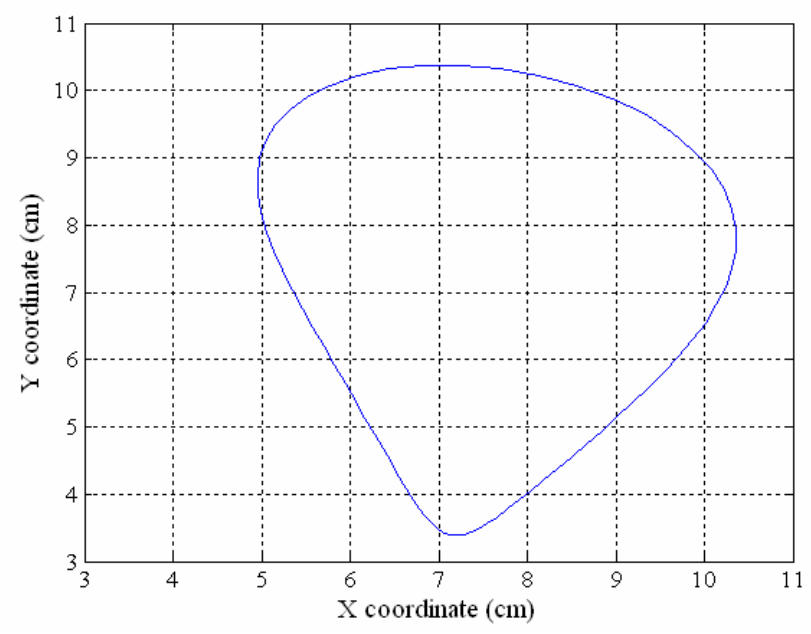

Fig. 8. Prescribed path for Example 2. 
Table 2. Coordinates of the precision point for Example 2 (cm).

\begin{tabular}{|c|c|c|c|c|c|c|c|c|c|c|c|c|c|}
\hline Point $n$ & 1 & 2 & 3 & 4 & 5 & 6 & 7 & 8 & 9 & 10 & 11 & 12 & 13 \\
\hline $\mathrm{X}$-coordinate & 5.52 & 5.77 & 6.08 & 6.44 & 6.83 & 7.25 & 7.69 & 8.13 & 8.56 & 8.95 & 9.31 & 9.62 & 9.87 \\
\hline Y-coordinate & 9.91 & 10.08 & 10.22 & 10.32 & 10.37 & 10.37 & 10.33 & 10.23 & 10.08 & 9.89 & 9.65 & 9.39 & 9.12 \\
\hline Point n & 14 & 15 & 16 & 17 & 18 & 19 & 20 & 21 & 22 & 23 & 24 & 25 & 26 \\
\hline X-coordinate & 10.07 & 10.22 & 10.31 & 10.35 & 10.35 & 10.31 & 10.24 & 10.14 & 10.01 & 9.87 & 9.71 & 9.54 & 9.37 \\
\hline Y-coordinate & 8.82 & 8.52 & 8.22 & 7.93 & 7.64 & 7.35 & 7.08 & 6.81 & 6.55 & 6.30 & 6.05 & 5.82 & 5.59 \\
\hline Point n & 27 & 28 & 29 & 30 & 31 & 32 & 33 & 34 & 35 & 36 & 37 & 38 & 39 \\
\hline X-coordinate & 9.19 & 9.02 & 8.84 & 8.66 & 8.50 & 8.33 & 8.18 & 8.04 & 7.90 & 7.77 & 7.66 & 7.55 & 7.45 \\
\hline Y-coordinate & 5.36 & 5.15 & 4.94 & 4.74 & 4.55 & 4.37 & 4.20 & 4.04 & 3.90 & 3.77 & 3.66 & 3.57 & 3.49 \\
\hline Point n & 40 & 41 & 42 & 43 & 44 & 45 & 46 & 47 & 48 & 49 & 50 & 51 & 52 \\
\hline X-coordinate & 7.36 & 7.28 & 7.20 & 7.13 & 7.06 & 6.99 & 6.92 & 6.85 & 6.79 & 6.71 & 6.64 & 6.55 & 6.47 \\
\hline Y-coordinate & 3.43 & 3.40 & 3.38 & 3.39 & 3.42 & 3.48 & 3.56 & 3.66 & 3.77 & 3.92 & 4.08 & 4.26 & 4.46 \\
\hline Point n & 53 & 54 & 55 & 56 & 57 & 58 & 59 & 60 & 61 & 62 & 63 & 64 & 65 \\
\hline X-coordinate & 6.37 & 6.27 & 6.17 & 6.06 & 5.94 & 5.82 & 5.70 & 5.58 & 5.46 & 5.34 & 5.24 & 5.14 & 5.06 \\
\hline Y-coordinate & 4.67 & 4.90 & 5.14 & 5.39 & 5.65 & 5.92 & 6.19 & 6.47 & 6.75 & 7.04 & 7.33 & 7.61 & 7.89 \\
\hline Point n & 66 & 67 & 68 & 69 & 70 & 71 & 72 & 73 & & & & & \\
\hline X-coordinate & 4.99 & 4.96 & 4.95 & 4.98 & 5.04 & 5.15 & 5.31 & 5.51 & & & & & \\
\hline Y-coordinate & 8.18 & 8.45 & 8.73 & 8.99 & 9.24 & 9.48 & 9.71 & 9.91 & & & & & \\
\hline
\end{tabular}

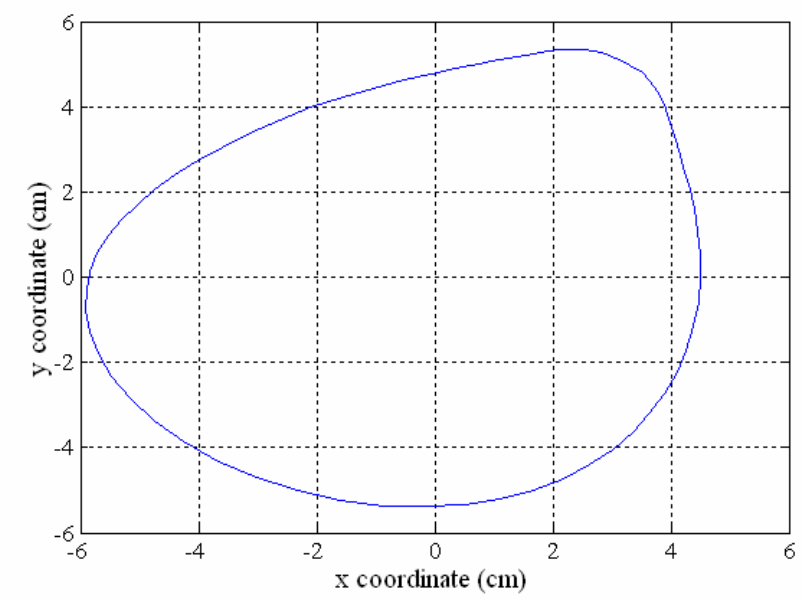

Fig. 9. Generated cam profile for Example 2.

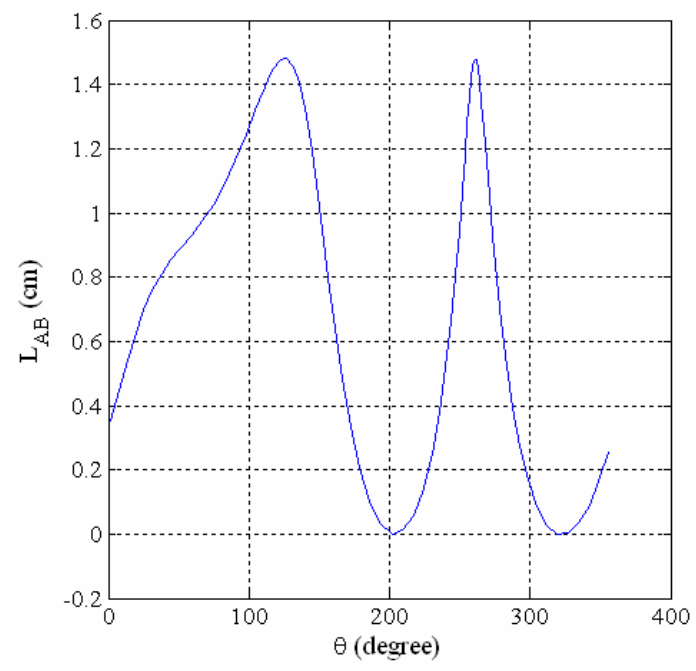

Fig. 10 The displacement of link 4 (follower) for example 2 


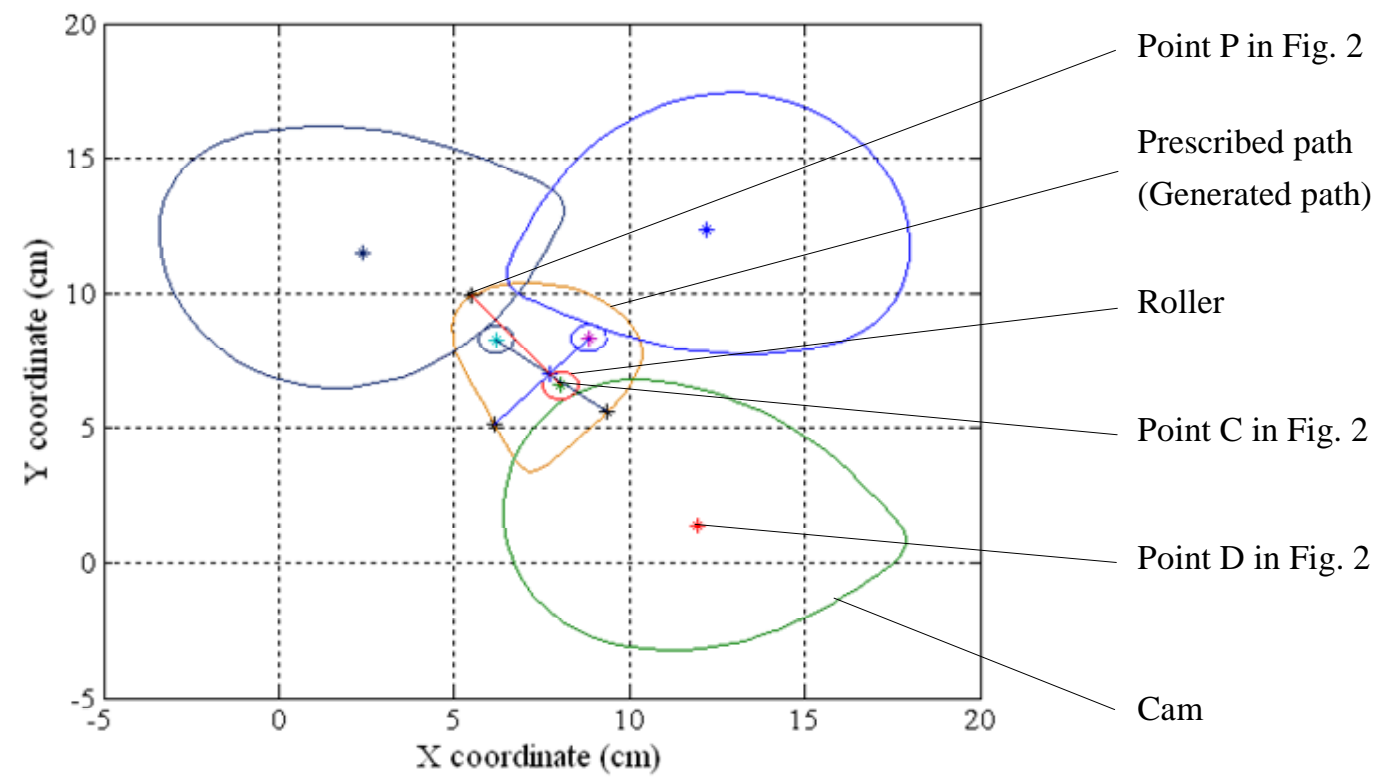

Fig. 11. Configurations corresponding to three precision points for Example 2.

Table 3. Design variables for Example 2 in centimeters $(\mathrm{cm})$.

\begin{tabular}{ccccccc}
\hline$r_{c}$ & $r_{4}$ & $r_{f}$ & $x_{A}$ & $y_{A}$ & $r_{p \max }$ & $r_{p \min }$ \\
\hline 7 & 2 & 0.5 & 7.71 & 6.99 & 3.66 & 2.17 \\
\hline
\end{tabular}

The results of this example also show that the shape and size of the generated cam profile were very similar to the prescribed path and satisfied the condition of a maximum pressure angle less than $30^{\circ}$. The results of this example also suggest that the proposed mechanism is suitable for generating diverse types of continuous curve paths.

\section{Example 3.}

In this example, we will generate a prescribed figure-eight path without time prescription, as shown in Fig. 12, also studied by Mundo et al.(2006). In their study, they synthesized a cam-linkage mechanism that combined a linkage mechanism and two disk-cam mechanisms, as shown in Fig. 13. The coordinate values of Point A, $\left(x_{A}, y_{A}\right)$, the maximum and minimum distances between Point A and points on the prescribed path, $\left(r_{p \max }, r_{p \min }\right)$, and the values of $r_{c}, r_{4}$, and $r_{f}$ are shown in Table 4. Figures 14-16 show the profile of the generated cam, the displacement of link4 (follower) and configurations of the proposed mechanism corresponding to the prescribed path, respectively.

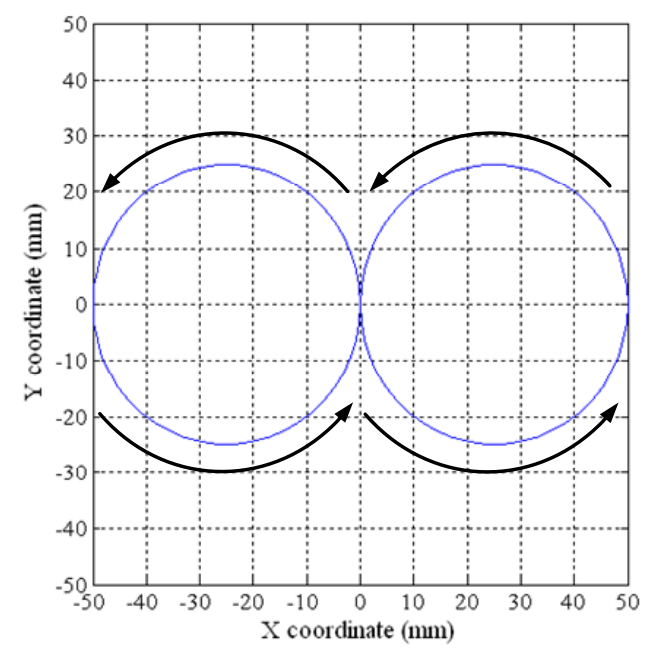

Fig. 12. Prescribed path for Example 3. 


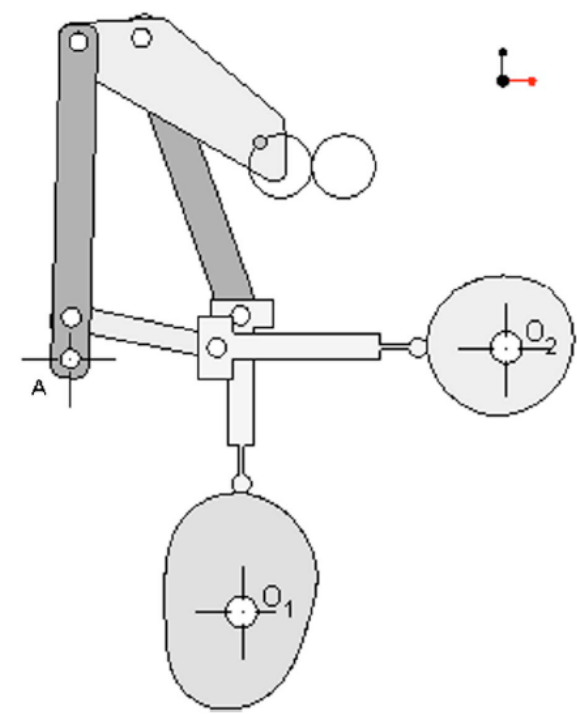

Fig. 13. Cam-linkage mechanism synthesized by Mundo et al.(2006) to generate the path shown in Fig. 12.

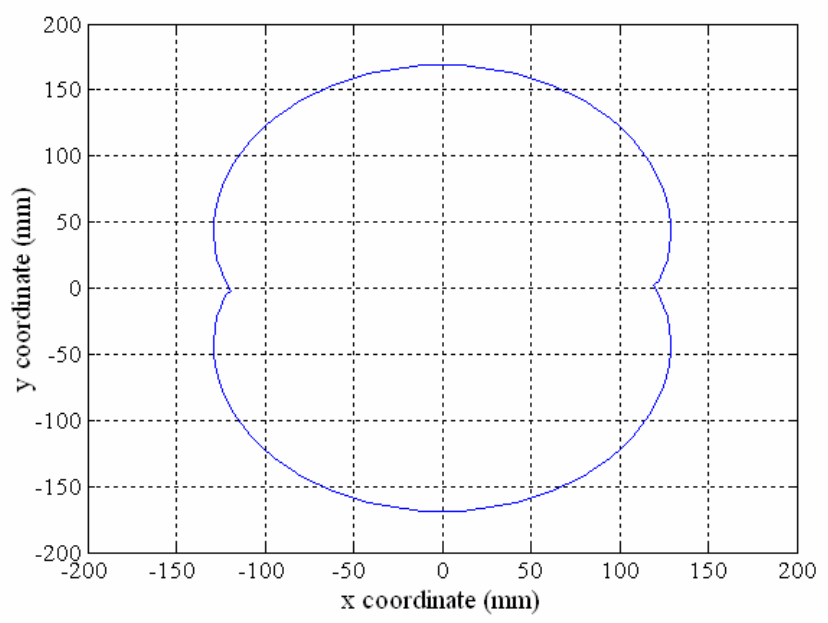

Fig. 14. Generated cam profile for Example 3.

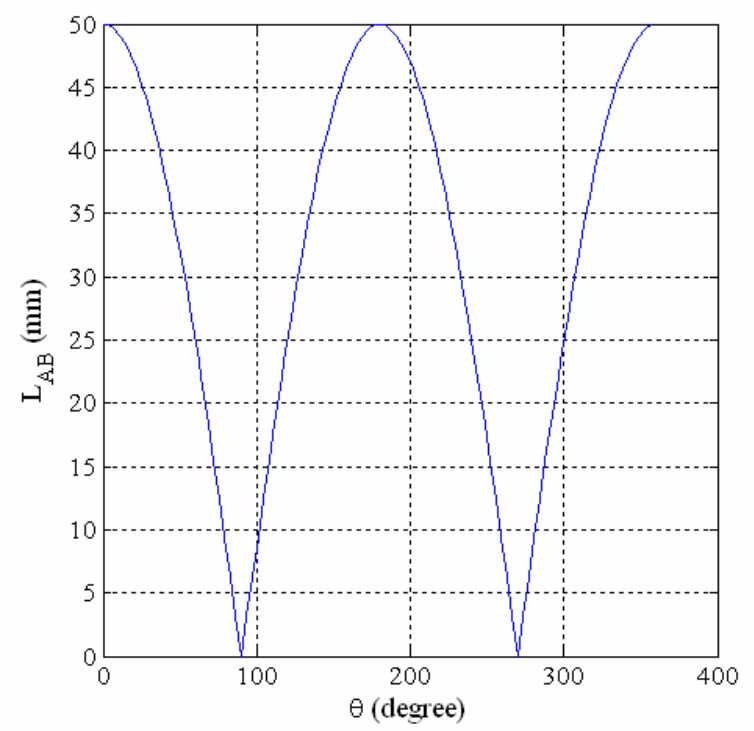

Fig. 15 The displacement of link 4 (follower) for example 2 


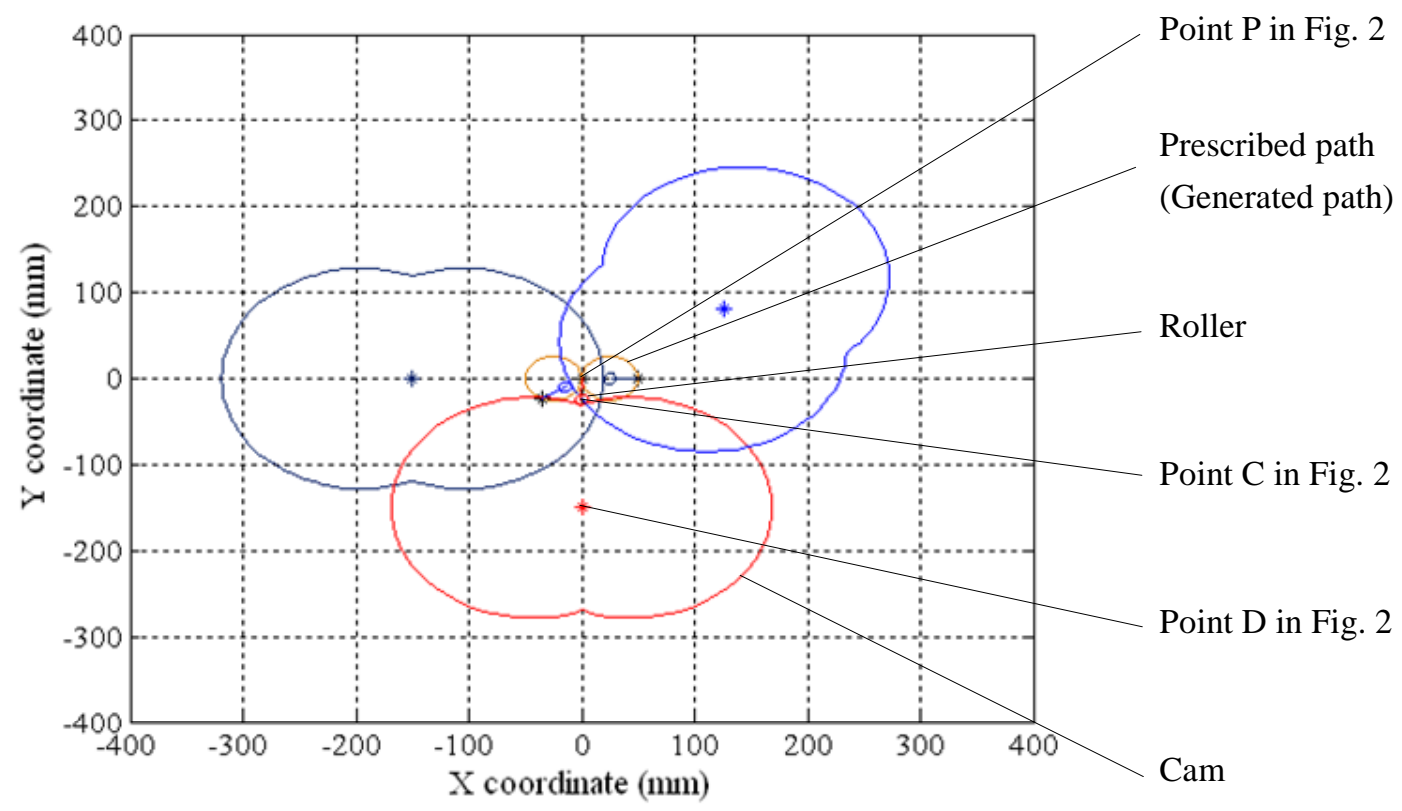

Fig. 16. Configurations corresponding to three precision points for Example 3.

Table 4. Design variables for Example 3 in millimeters (mm).

\begin{tabular}{ccccccc}
\hline$r_{c}$ & $r_{4}$ & $r_{f}$ & $x_{D}$ & $y_{D}$ & $r_{p \max }$ & $r_{p \min }$ \\
\hline 125 & 10 & 5 & 0 & 0 & 50 & 0 \\
\hline
\end{tabular}

The results of this example show the feasibility of obtaining a cam profile with maximum pressure angles of less than $30^{\circ}$ by selecting a suitable valve of $r_{c}$. The results of this example also suggest that the proposed mechanism is suitable for generating symmetric paths with an intersection.

According to the results of these examples, several conclusions can be drawn, using the method described in Ref. [20] for comparison. The advantages of the proposed method are its simplicity in its design, and its simple, compact structure for generating diverse types of exact continuous curve paths. The proposed method is suitable for generating symmetric paths with an intersection as well.

\section{Conclusion}

A new cam-geared mechanism was proposed for exact path generation, consisting of a cam-follower mechanism and an elementary planetary gear train. The formation and mobility of the proposed mechanism were illustrated in detail. A new design method and process for exact path generation were presented. The generated path of the proposed mechanism exactly passed through all points on the prescribed path. The advantages of the proposed method are its simplicity, the simple, compact structure of the mechanism, and no numbers limitation of precision point. This new mechanism is suitable for generating diverse continuous curve paths and symmetric curve paths with an intersection. Three examples are provided to show the feasibility and effectiveness of this proposed method.

\section{Acknowledgements}

The author is grateful to the Ministry of Science and Technology of the Republic of China (Taiwan, R.O.C.) for their support of this research under grant NSC 102-2221-E-224-003-MY2.

\section{References}

Angeles, J., Alivizators, A. and Akhras, A., An unconstrained nonlinear least-square method of RRRR planar path generators, Mechanism and Machine Theory, Vol. 23, No. 5 (1988), pp.343-353.

Buskiewicz, J., Use of shape invariants in optimal synthesis of geared five-bar linkage, Mechanism and Machine 
Theory, Vol. 45, No. 2 (2010), pp.273-290.

Chuenchom, T. and Kota, S., Synthesis of programmable mechanisms using adjustable dyads, ASME journal of Mechanical Design, Vol. 29, No. 5 (1997), pp.232-237.

Cabrera, J. A., Simon, A. and Prado, M., Optimal synthesis of mechanisms with genetic algorithms, Mechanism and Machine Theory, Vol. 37, No. 10 (2002), pp.1165-1177.

Chanekar, P. V. and Ghosal, A., Optimal synthesis of adjustable planar four-bar crank-rocker mechanisms for approximate multi-path generation, Mechanism and Machine Theory, Vol. 69, (2013), pp.263-277.

Gatti, G. and Mundo, D., Optimal synthesis of six-bar cammed-linkages for exact rigid-body guidance, Mechanism and Machine Theory, Vol. 42, No. 9 (2007), pp.1069-1081.

Key, F. J. and Haws R. E., Adjustable mechanisms for exact path generation, ASME Journal of Engineering for Industry, Vol. 92, No. 7 (1975), pp.702-707.

Laribi, M.A., Milka, A., Romdhance, L. and Zeghloul, S., A combined genetic algorithm-fuzzy logic method (GA-FL) in mechanism synthesis, Mechanism and Machine Theory, Vol. 39, No. 7 (2004), pp.717-735.

Lin, W. Y., A GA-DE hybrid evolutionary algorithm for path synthesis of four-bar linkage, Mechanism and Machine Theory, Vol. 45, No. 8 (2010), pp.1096-1107.

Mundo, D., Lio, J. Y. and Yan, H. S., Optimal synthesis of cam-linkage mechanisms for precise path generation, ASME Journal of Mechanical Design, Vol. 128, No. 6 (2006), pp.1253-1260.

Mundo, D., Gatti, G. and Dooner, D. B., Optimized five-bar linkages with non-circular gears for exact path generation, Mechanism and Machine Theory, Vol. 44, No. 4 (2009), pp.751-760.

Matekar, S. B. and Gogate, G. R., Optimum synthesis of path generating four-bar mechanisms using differential evolution and a modified error function, Mechanism and Machine Theory, Vol. 52 (2012), pp.158-179.

Peng, C. and Sodhi, R. S., Optimal synthesis of adjustable mechanisms generating multi-phase approximate paths, Mechanism and Machine Theory, Vol. 45, No. 7 (2010), pp.989-996.

Russell, K. and Sodhi, R. S., On the design of slider-crank mechanisms. Part II: multi-phase path and function generation, Mechanism and Machine Theory, Vol. 40, No. 3 (2005), pp.301-317.

Singh, Y.P. and Kohli, D., Synthesis of cam-link mechanisms for exact path generation, Mechanism and Machine Theory, Vol. 16, No. 4 (1981), pp.447-457.

Shimojima, H., Ogawa, K. and Sato O., Kinematic synthesis of adjustable mechanisms, Bulletin of JSME, Vol. 26 , No. 214 (1983), pp.627-632.

Sancibrian, R., Garcia, P., Viadero, F. and Fernandez, A., A general procedure based on exact gradient determination in dimensional synthesis of planar mechanisms, Mechanism and Machine Theory, Vol. 41, No. 2 (2006), pp.212-229.

Soong, R. C. and Wu, S. L., Design of variable coupler curve four-bar mechanisms, Journal of the Chinese Society of Mechanical Engineers, Vol. 30, No. 3 (2009), pp.249-257.

Soong, R. C. and Chang, S. B., Synthesis of function-generation mechanisms using variable length driving links, Mechanism and Machine Theory, Vol. 46, No. 11 (2011), pp.1669-1706.

Soong, R. C., On the design of new programmable exact path generators, Transactions of the Canadian Society for Mechanical Engineering, Vol. 37, No. 3 (2013), pp.685-692.

Ullah, I. and Kota, S., Optimal synthesis of mechanisms for path generation using Fourier descriptors and global search methods, ASME Journal of Mechanical Design, Vol. 119, No. 4 (1997), pp.504-510.

Vasiliu, A. and Yannou, B., Dimensional synthesis of planar mechanisms using neural networks: application to path generator linkages, Mechanism and Machine Theory, Vol. 36, No. 2 (2001), pp.299-310.

Ye, Z. and Smith, M. R., Design of a combined cam-linkage mechanism with an oscillating roller follower by an analytical method, Proc. IMechE Part C: Journal of Mechanical Engineering Science, Vol. 219, No. 4 (2005), pp.419-427.

Zhou, H. and Cheung, Edmund. H.M.,Optimal synthesis of crank-rocker linkages for path generation using the orientation structural error of the fixed link, Mechanism and Machine Theory, Vol. 36, No. 8 (2001), pp.973-982.

Zhou, H. and Ting, K.L., Adjustable slider-crank linkages for multiple path generation, Mechanism and Machine Theory, Vol. 37, No. 5 (2002), pp.499-509.

Zhou, H., Dimensional synthesis of adjustable path generation linkages using the optimal slider adjustment, Mechanism and Machine Theory, Vol. 44, No. 10 (2009), pp.1866-1876. 